\title{
Evidence of dipstick superiority over urine microscopy analysis for detection of hematuria
}

\author{
Aurélien Bataille1, Morgane Wetzstein ${ }^{1}$, Alexandre Hertig 1,2,4, Sophie Vimont ${ }^{1,3,4}$, Eric Rondeau 1,2,4 \\ and Pierre Galichon ${ }^{1,2,4^{*}}$ (B)
}

\begin{abstract}
Background: There is an unresolved debate on the best screening method for hematuria as a symptom of glomerulonephritis or urological malignancies. The urinary dipstick is generally considered as an imperfect surrogate for urine microscopy analysis.

Results: We designed a study to compare urine microscopy analysis, urinary dipstick and flow cytometry, using controlled dilutions of blood in urine samples from volunteers collected in two different physiologically-relevant conditions (basal state and hyperhydration). We found that although all techniques were $100 \%$ effective in detecting hematuria at basal state, these results were variably reproduced when testing the same final amount of hematuria in urine collected after hyperhydration. Our data shows a variable sensitivity for the detection of hematuria by urine microscopy analysis or flow cytometry, but not by urinary dipstick.
\end{abstract}

Conclusions: Urinary dipstick qualifies as a better screening test for hematuria than urine microscopy analysis or flow cytometry, as it is sensitive and performs better in unstandardized conditions. It is universally available and also faster and cheaper than cytometric techniques.

Keywords: Screening test, Hematuria, Sensitivity

\section{Findings}

\section{Background}

Screening for hematuria is recommended in many situations, including screening of high risk patients in occupational medicine [1-3] and tests are expected to be sensitive. Missed or delayed diagnosis of hematuria may lead to neglecting urological (cancer) or nephrological (glomerulonephritis) life-threatening diseases [4]. There has long been a debate as to whether urine dipstick or urine microscopy analysis (UMA) is the preferred testing method, but there is no consensus amongst practitioners $[5,6]$. UMA performance may be altered by red blood cell (RBC) degradation in urine, and urine dipstick may

\footnotetext{
*Correspondence: pierre.galichon@aphp.fr

2 Urgences Néphrologiques et Transplantation Rénale, Hôpital Tenon, 4, rue de la Chine, 75020 Paris, France

Full list of author information is available at the end of the article
}

misdiagnose hemoglobinuria or myoglobinuria as hematuria [6]. To our knowledge, no controlled study has compared urine dipstick and UMA to an independent gold standard, and studies evaluating the urine dipstick using UMA as a benchmark do not evaluate the diagnostic performance of UMA itself. Our objective was to compare urine dipstick and UMA as a diagnostic test for hematuria, using urine samples with a known concentration of RBC.

\section{Methods}

We obtained a calibration by diluting a controlled amount of blood in urine. Control urine was collected from volunteers (healthy young males to avoid genital blood contamination) after informed oral consent. $50 \mathrm{~mL}$ midstream urine was collected at baseline and after $1.5 \mathrm{~L}$ of water ingestion. Blood was taken from one volunteer, diluted in urine to $1: 10^{3}$, and ten fold serial dilutions in 
urine were performed up to $1: 10^{8}$. Urine dipstick (Multistix ${ }^{\circledR}$ 8SG, Siemens) with an automatic analyser (Clinitek Status $\left.{ }^{\circledR}\right)$ yielded semi-quantitative results $(0$, prints, + , ,+++++ , Additional file 1). Urine microscopic analysis at a 400-fold optical magnification (Leitz Wetzlar ${ }^{(B)}$ Ortholux) in $1 \mu \mathrm{L}$ counting chambers allowing quantitative per volume assessment of hematuria [7] and flow cytometry (MACSQuant ${ }^{\circledR}$ Analyser, Miltenyi Biotec) gating $\mathrm{RBC}$ on a side scatter versus forward scatter plot yielded a count $/ \mathrm{mm}^{3}$ (Additional files 2 and 3 respectively). Data are summarized as median and interquartile range. Two-sided Wilcoxon tests were performed at 0.05 significance level, and paired when appropriate using $\mathrm{R}$ statistical package (version 2.14.1).

Using serial dilutions, we found that urine dipstick, UMA and flow cytometry detected hematuria at the threshold of $1: 10^{6}$ blood dilution, with the greatest sensitivity for flow cytometry (100\%), followed by urine dipstick (83\%) and UMA (64\%). At the 1:10 ${ }^{5}$ dilution, all three techniques had $100 \%$ sensitivity (Fig. 1), and we thus decided to use this dilution to compare the robustness of the three different test depending on hydration.

\section{Results}

We collected urine after water ingestion to evaluate the three methods in a different clinical setting and to compare to baseline control urine. As expected, we found that urine density decreased after water load [1010 (1006; 1010) vs. 1030 (1022; 1030), $p=0.034$ ]. When we assessed the three different methods in baseline and hypotonic urine, using the same $1: 10^{5}$ dilution, we found that the cytomorphological tests (UMA and flow cytometry) were not reproducible, with a

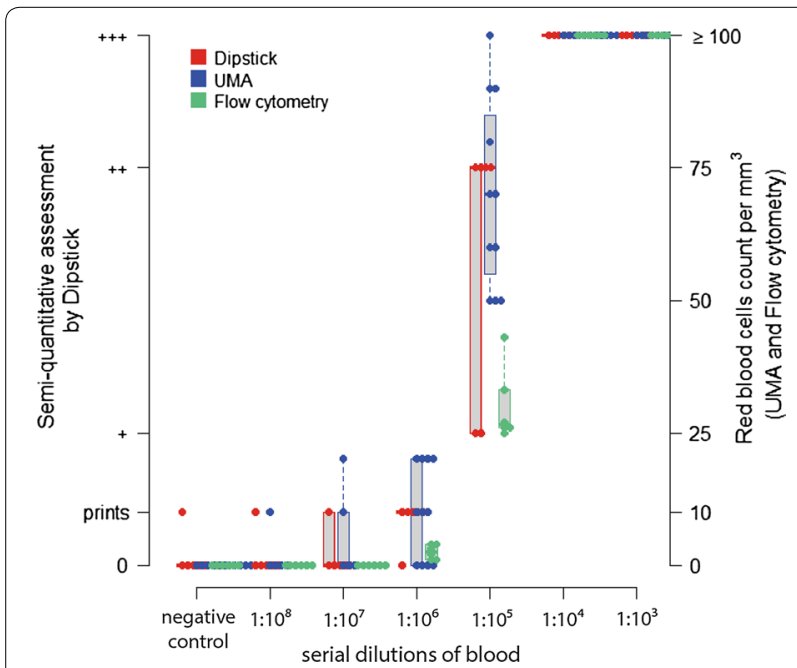

Fig. 1 Detection of hematuria by urine dipstick, UMA or flow cytometry at increasing concentrations of RBC systematic underestimation of hematuria $(57 \%$ median fold decrease, $\mathrm{p}=0.036$ and $92 \%$ median fold decrease, $\mathrm{p}=0.031$, respectively) whereas assessment by the urine dipstick conserved its sensitivity (++ positivity) (Fig. 2).

We compared the sensitivity of three different methods for diagnosing hematuria, and the robustness of these methods when there is a clinically relevant change in urine composition. Urine dipstick was more sensitive than UMA, especially in hypotonic urine. The enhanced sensitivity of the dipstick is explained by its ability to detect heme even after RBC lysis. We performed the blood dilutions with urine collected at basal state and after hydration to ensure that the chemical and physical conditions were relevant to patients with hematuria. This is very relevant to urological hematuria (i.e. a bleeding occurring directly in urine), and perhaps less to nephrological hematuria (where the RBC journey with primary urine in the tubules). We chose to evaluate the detection of hematuria independently of the effect of changes in diuresis, but this parameter could enhance the effect of hydration that we observed: in patients, hypotonic urine is often associated with increased diuresis and urine dilution could further decrease the sensitivity of hematuria detection. The dipstick can be performed immediately, but UMA needs to be brought to the laboratory first, and delay also reduces the sensitivity of UMA [8].

\section{Conclusions}

Thus, instead of considering the urine dipstick as a surrogate marker for UMA, it should be the preferred method for screening hematuria. Timed urine samples were once considered to be the gold standard, but UMA is now recommended by most guidelines. We believe that UMA may not be reliable if performed in unstandardized

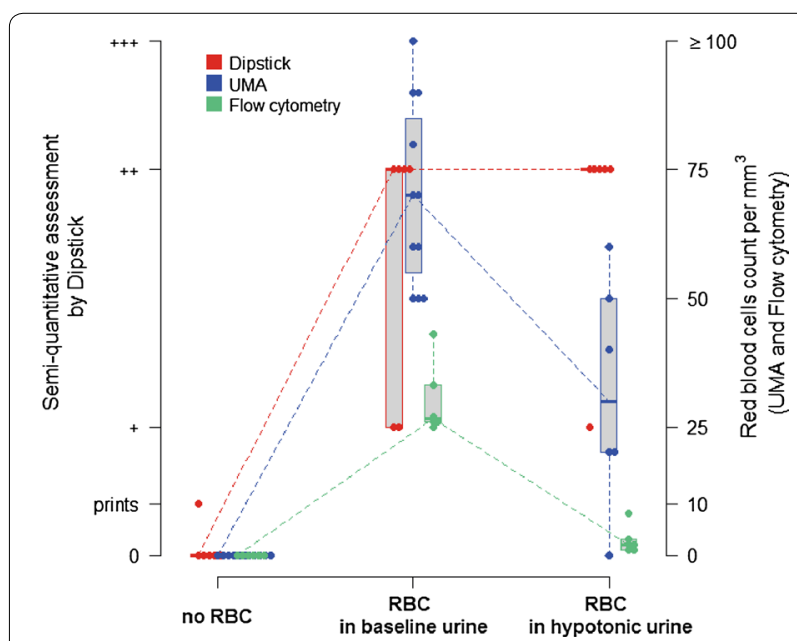

Fig. 2 Results of UMA, urine dipstick and flow cytometry for hematuria in baseline and hypotonic urine 
conditions, and that it should rather be a confirmatory test performed in controlled conditions (midstream urine harvested in the morning when it is more acid and hypertonic), to rule out isolated pigmenturia and to detect dysmorphic RBC. The urine dipstick should, therefore, be preferred for the screening of hematuria as it is more sensitive and cheaper than UMA.

\section{Additional files}

Additional file 1. BU2.csv contains the dipstick data. "nom" stand for individual numbers, "H" for basal morning urine, "h" for urine after hydration, "densité" for urine density, and "10 px" for the $10^{x}$ red blood cell concentrations.

Additional file 2. ECBU2.csv contains the microscopy analysis data. "nom" stand for individual numbers, "H" for basal morning urine, "h" for urine after hydration, "densité" for urine density, and "10 px" for the $10^{x}$ red blood cell concentrations.

Additional file 3. FACS2.cSv contains the flow cytometry data. "nom" stand for individual numbers, "H" for basal morning urine, "h" for urine after hydration, "densité" for urine density, and "10 px" for the $10^{x}$ red blood cell concentrations.

\section{Abbreviations}

UMA: urine microscopy analysis; RBC: red blood cells.

\section{Authors' contributions}

AB designed the study, collected the data, performed the statistical analysis and drafted the manuscript. MW collected the data. SV participated to the design of the study and urine microscopy analyses. AH and ER participated to the analysis of the results. PG conceived and designed the study and collected the data. All authors read and approved the final manuscript.

\section{Author details}

${ }^{1}$ Inserm UMR_S 1155, Hôpital Tenon, Paris, France. ${ }^{2}$ Urgences Néphrologiques et Transplantation Rénale, Hôpital Tenon, 4, rue de la Chine, 75020 Paris,
France. ${ }^{3}$ Service de Bactériologie, Hôpital Tenon, Paris, France. ${ }^{4}$ Université Pierre et Marie Curie (Paris 6), Sorbonne Universités, Paris, France.

\section{Acknowledgements}

There was no funding for this study.

\section{Competing interests}

The authors declare that they have no competing interests.

\section{Availability of data and materials}

The raw data can be accessed in the online additional supporting files.

\section{Consent for publication}

We obtained oral consent before collecting urine. In compliance with the French and international legislation, the need for approval of this non-invasive, non-interventional research was waived by our Institutional Review Board (Comité de Protection des Personnes lle de France V, ref e-7-16).

Received: 4 May 2016 Accepted: 26 August 2016

Published online: 08 September 2016

References

1. Brown RS. Has the time come to include urine dipstick testing in screening asymptomatic young adults? JAMA. 2011;306:764-5.

2. Fradet $Y$. Screening for bladder cancer: the best opportunity to reduce mortality. Can Urol Assoc J. 2009;3:S180-3.

3. Pashayan N, Khogali M, Major SC. Routine urinalysis of patients in hospital in Lebanon: how worthwhile is it. J Med Screen. 2002;9:181-6.

4. Vivante A, Afek A, Frenkel-Nir Y, et al. Persistent asymptomatic isolated microscopic hematuria in Israeli adolescents and young adults and risk for end-stage renal disease. JAMA. 2011;306:729-36.

5. Moore GP, Robinson M. Do urine dipsticks reliably predict microhematuria? The bloody truth! Ann Emerg Med. 1988;17:257-60.

6. Rao PK, Gao T, Pohl M, Jones JS. Dipstick pseudohematuria: unnecessary consultation and evaluation. J Urol. 2010;183:560-4.

7. European COLM. European urinalysis guidelines. Scand J Clin Lab Invest Suppl. 2000;231:1-86.

8. Silkensen JR, Kasike BL. Laboratory assessment of renal disease: clearance, urinalysis, and renal biopsy. In: Brenner BM, editor. The kidney. 7th ed. Philadelphia: Saunders; 2004. p. 1107-50.

\section{Submit your next manuscript to BioMed Central and we will help you at every step: \\ - We accept pre-submission inquiries \\ - Our selector tool helps you to find the most relevant journal \\ - We provide round the clock customer support \\ - Convenient online submission \\ - Thorough peer review \\ - Inclusion in PubMed and all major indexing services}

- Maximum visibility for your research

Submit your manuscript at www.biomedcentral com/submit
Ciomed Central 